\title{
Hennekam Syndrome: A Case Report
}

\author{
Yeong Guk Lee, MD, Seung Chan Kim, MD, Si-Bog Park, MD, PhD, Mi Jung Kim, MD, PhD \\ Department of Rehabilitation Medicine, Hanyang University College of Medicine, Seoul, Korea
}

\begin{abstract}
Hennekam syndrome is a rare autosomal recessive disorder resulting from malformation of the lymphatic system. The characteristic signs of Hennekam syndrome are lymphangiectasia, lymph edema, facial anomalies, and mental retardation. This is a case in which a patient presented with left-arm lymphedema, facial-feature anomalies, and multiple organ lymphangiectasia consistent with symptoms of Hennekam syndrome. There is no curative therapy at this time, but rehabilitative treatments including complete decongestive therapy for edema control appeared to be beneficial.
\end{abstract}

Keywords Lymphedema, Hennekam lymphangiectasia lymphedema syndrome

\section{INTRODUCTION}

Hennekam syndrome is an autosomal recessive disorder resulting from malformation of the lymphatic system. This syndrome was first described by Dutch physician Hennekam in 1989 [1]. It is a very rare syndrome and fewer than 50 cases have been reported in medical literature [2].

The characteristic signs of Hennekam syndrome are lymphangiectasia, lymph edema, facial anomalies, and mental retardation [3]. Malformations of lymphatic channels block the lymph circulation, and accumulation of fluids affects multiple body parts including the face and limbs, as well as internal organs. Facial features are characterized by a flattened appearance of the face, a broad depressed nasal bridge, hypertelorism, epicanthal folds, a small mouth, and other anomalies [3].
This syndrome is often diagnosed on the basis of clinical symptoms, but more recent studies have been made through genetic analysis. About $25 \%$ of patients who have Hennekam syndrome were found to have autosomal recessive mutations in CCBE1 [4] and a further $20 \%$ of patients have mutations in FAT4 [5].

We present the case of a 28-month-old Russian female affected with Hennekam syndrome, who was previously undiagnosed.

\section{CASE REPORT}

A 28-month-old Russian female presented with congenital left-arm edema. She was born after 38 weeks' gestation by vaginal delivery, and her birth weight was 2.95 $\mathrm{kg}$. She was the second of fraternal twins, but her twin sister displayed no abnormalities. The parents had no 
history of radiation exposure, the mother had no major illness during pregnancy or abnormal obstetric history. No other family members had similar phenotypic features. She was admitted to a neonatal intensive care unit (NICU) in Russia for treatment of respiratory difficulty and for intense evaluation for 1 month, but no diagnosis was made, and she was eventually referred to our hospital.

At the time of admission, the patient was conscious and her vital signs were within normal limits. Her height was $94 \mathrm{~cm}$ (90th centile according to the Center for Disease Control and Prevention [CDC] growth chart) and weight was $14 \mathrm{~kg}$ (between 75th-90th centile per the CDC growth chart). She had significant left-arm edema (without pain), limitation of motion, and difficulty with arm movement (Fig. 1). The circumferences of right and left arms were as

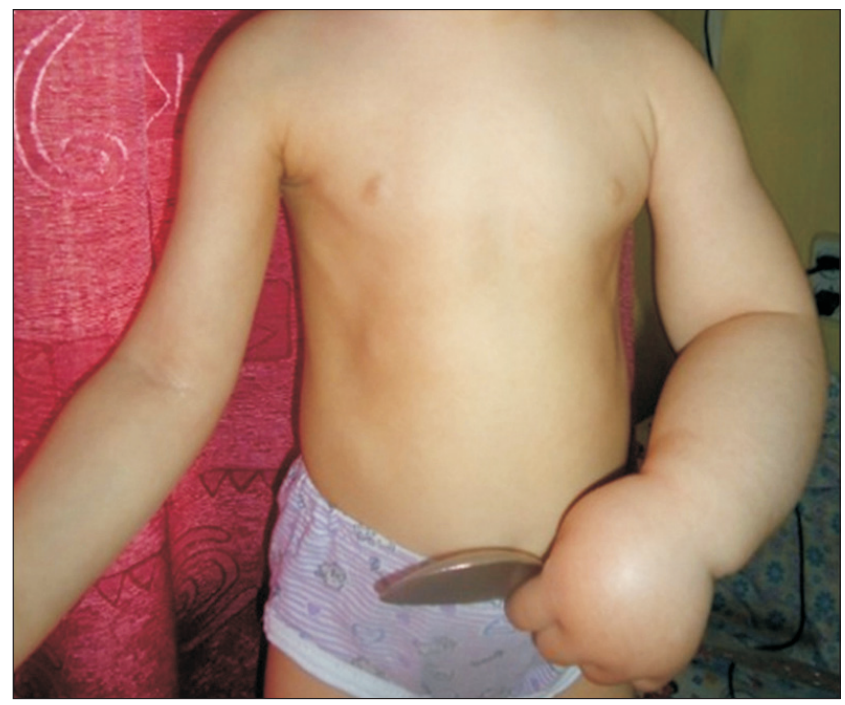

Fig. 1. Lymph edema of left upper extremity. follow: upper arm (midpoint between the tip of the olecranon process and the tip of the acromion), $14.5 \mathrm{~cm}$ and $22 \mathrm{~cm}$; forearm (at two fingers distance the elbow crease), $14 \mathrm{~cm}$ and $22.5 \mathrm{~cm}$, respectively. The amount of water and moisture ratio (i.e., extracellular fluid/total water amount) in the right upper limb was $420 \mathrm{~mL}$ and $0.389 \%$, respectively, and those in the left upper limb were 1,050 $\mathrm{mL}$ and $0.412 \%$, respectively in bioimpedance analysis (InBody S10; Biospace, Seoul, Korea). A dysmorphic face with flat midface, hypertelorism, depressed nasal bridge, a bulbous nasal tip, and epicanthal folds was also evident (Fig. 2). All other parts of the body were normal in appearance.

Various rehabilitative assessment tools were applied, with the help of a Russian interpreter, to evaluate the patient's development. The results showed some developmental delay in gross motor skills, fine motor skills, personal-social functions, and functional activities. Routine blood tests and urinalysis were within normal limits. Total protein $(6.1 \mathrm{~g} / \mathrm{dL})$ and albumin $(4.0 \mathrm{~g} / \mathrm{dL})$ levels were also within normal limits.

Based on the characteristic dysmorphic face with congenital lymphatic edema, the possibility of Hennekam syndrome was considered. To identify lymphatic malformations, which were additional typical features of Hennekam syndrome, we used various diagnostic evaluations including X-ray, computed tomography (CT), angiography, lymphatic scan, brain magnetic resonance imaging (MRI), chest CT, and abdomen ultrasonography (US), which indicated lymphatic malformation of multiple organs (Fig. 3). We diagnosed Hennekam syndrome, based on the clinical features of these various lymphatic abnormalities.

The patient received 7 daily sessions of comprehen-
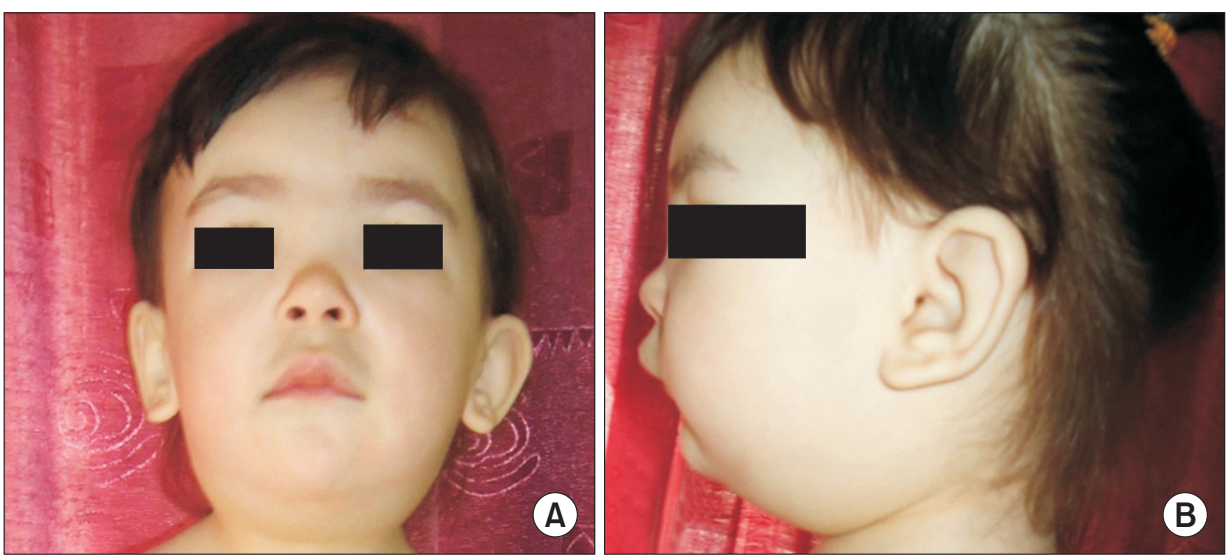

Fig. 2. Dysmorphic face with flat midface, hypertelorism, and broad nasal bridge of front view (A) and side view (B). 

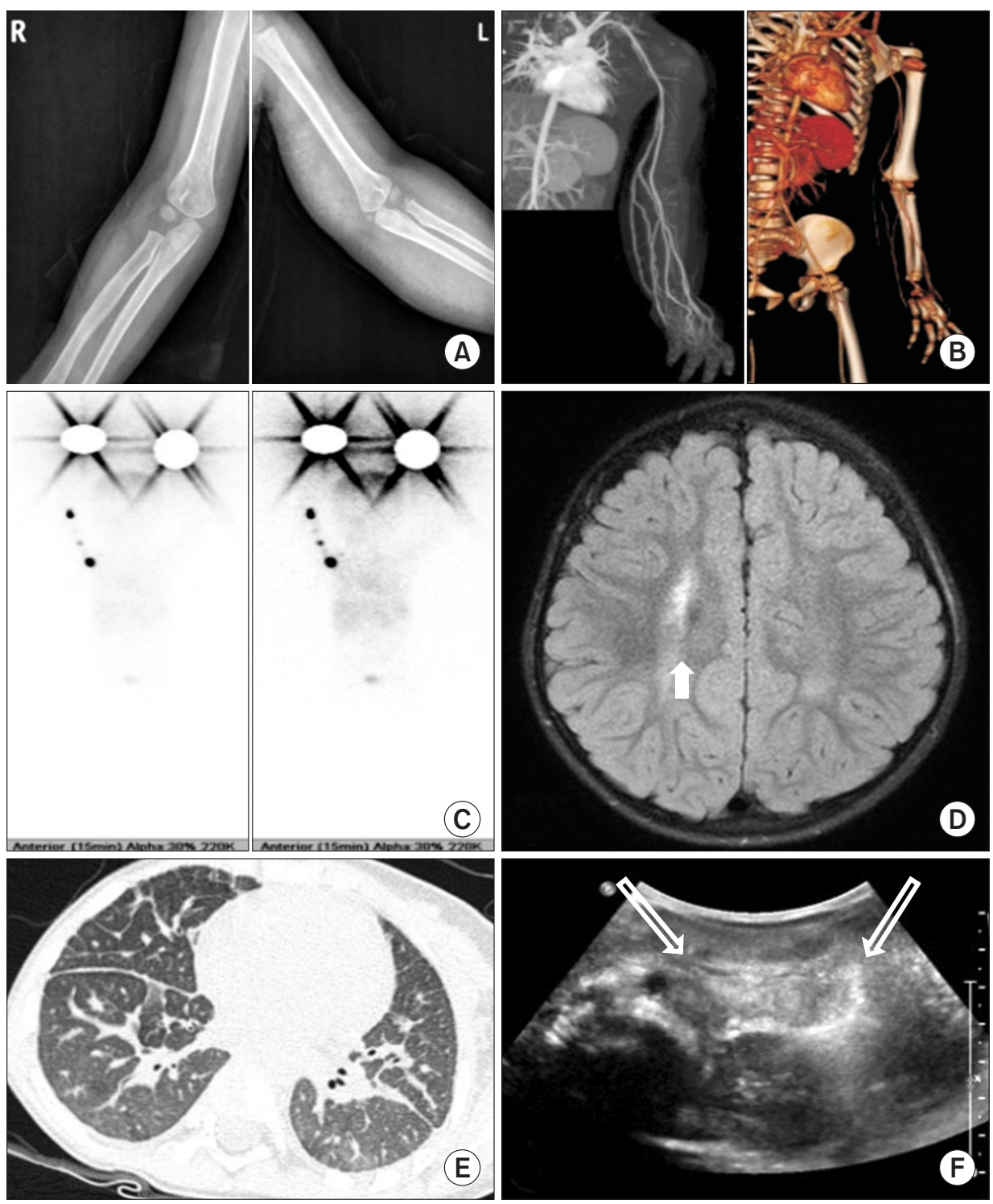

Fig. 3. On diagnostic evaluation of multiple organs, (A) the simple $\mathrm{X}$-ray of both upper extremities showed no gross bony abnormality. (B) The computed tomography (CT) angiography showed a marked lymphedema in the left upper extremity. (C) The lymphatic scan showed no visible lymphatic flow of left upper extremity, which implies primary lymphedema. (D) The brain MRI showed a focal increased signal intensity (arrow). (E) The chest CT showed lymphangiectasia of both lungs. (F) The abdomen ultrasonography showed lymphangiectasia in small bowel mesentery (open arrow). sive rehabilitative treatment, including activities of daily living training, low-level laser therapy (10-minute application with a wavelength range of $600-1,000 \mathrm{~nm}$ ), and complete decongestive therapy for lymphedema of left arm. The complete decongestive therapy included manual lymph drainage (10-minute sessions performed by a physical therapist), compression (a 4 -cm gauze bandage from distal hand to armpit and a $6-\mathrm{cm}$ bandage from wrist to armpit were applied for a minimum of 20 hours per day), and exercise (performed twice a day for $15 \mathrm{~min}$ utes while wearing compression bandages).

After 7 sessions of treatment, the changes in the left arm circumference were as follows: upper arm, from $22 \mathrm{~cm}$ to $19 \mathrm{~cm}$; forearm, from $22.5 \mathrm{~cm}$ to $21 \mathrm{~cm}$, respectively.
Bioimpedance analysis of the left upper limb showed a decrease in water retention from $1,050 \mathrm{~mL}$ to $980 \mathrm{~mL}$, and a decrease in moisture ratio from $0.412 \%$ to $0.405 \%$. Surgery was not performed because the lymphedema of this case was not severe enough to have significant functional limitations. Economic hardship forced the patient's return to Russia before further evaluations, including genetic analysis, and long-term rehabilitation could be performed.

We diagnosed a rare congenital lymphatic abnormality, Hennekam syndrome, and were able to demonstrate improvement in the patient's condition after only a brief period of rehabilitation treatment. 


\section{DISCUSSION}

In this case, we suspected Hennekam syndrome based on congenital left-arm edema and typical facial features. Lymphatic malformations of multiple organs were confirmed by CT angiography, lymphatic scan, chest CT, and abdominal US.

The facial features of Hennekam syndrome patients typically include a flattened appearance of the face, a flat and broad nasal bridge, hypertelorism, and epicanthal folds, as was the case for this patient [3]. Other facial characteristics such as low set and dysplastic ears, smooth philtrum, and dental anomalies were not evident in this case. The patient's facial features were similar to those observed in previous cases of Hennekam syndrome, and it was these facial features that led to the diagnosis.

The developmental abilities of patients with Hennekam syndrome have ranged from almost normal psychomotor development to varying levels of mental retardation [3]. Application of rehabilitative developmental assessment tools showed slightly delayed psychomotor development. Due to the young age of the patient, 28 months, periodic follow-up of psychomotor development is required.

Intestinal lymphangiectasia can lead to severe protein loss resulting in hypogammaglobulinemia, hypoalbuminemia, lymphopenia, and growth retardation [6]. It has been reported that growth retardation may be caused by both gastrointestinal protein loss and malabsorption [6]. Ultrasonographic findings of intestinal lymphangiectasia were found in this patient, but no protein-loss pattern or growth retardation pattern was observed.

The congenital pulmonary lymphangiectasis (CPL) is a rare developmental disorder of the pulmonary lymphatic vessels [7]. Early death of the affected newborn is usually caused by severe pulmonary hypoplasia [7]. A chest CT showed pulmonary lymphangiectasis in this patient, which may have been related to the NICU care at the time of birth. Pulmonary-related complications are directly related to survival, so regular follow-up chest X-rays or chest CTs are required.

About $33 \%$ of cases of Hennekam syndrome patients have seizures, varying from absences to tonic or atonic attacks [3]; however, this patient had never experienced a seizure. The patient's brain MRI showed focal increased signal intensity in right periventricular white matter, which implies gliotic change or periventricular leukomalacia.

Treatment will vary according to symptoms [3]. Some patients may require a high-protein, low-fat diet with medium-chain triglyceride supplementation in the case of protein-losing enteropathy [8]. The lymphedema may be severely disabling, but surgery [9] is considered a treatment of last resort. Surgery may improve limb size and restore some function in severe lymphedema that does not respond to conservative treatment.

The significance of this case report is that it is the first paper on rehabilitative evaluation and treatment for Hennekam syndrome. Also, to our knowledge, this is the first Russian case to be reported in Korea. The limitations of this case report are the lack of genetic testing for the patient and the lack of follow-up care of the patient.

In summary, the patient was diagnosed with Hennekam syndrome based on clinical phenotypic features such as lymphangiectasia, lymph edema, and facial anomalies. There is no curative therapy at this time, but rehabilitative treatments including complete decongestive therapy for edema control appeared to be beneficial.

\section{CONFLICT OF INTEREST}

No potential conflict of interest relevant to this article was reported.

\section{REFERENCES}

1. Hennekam RC, Geerdink RA, Hamel BC, Hennekam FA, Kraus P, Rammeloo JA, et al. Autosomal recessive intestinal lymphangiectasia and lymphedema, with facial anomalies and mental retardation. Am J Med Genet 1989;34:593-600.

2. Rao BS, Vani MS, Kanth BS. Hennekam lymphangiectasia syndrome. Int J Res Med Sci 2015;3:516-9.

3. Van Balkom ID, Alders M, Allanson J, Bellini C, Frank U, De Jong G, et al. Lymphedema-lymphangiectasiamental retardation (Hennekam) syndrome: a review. Am J Med Genet 2002;112:412-21.

4. Alders M, Hogan BM, Gjini E, Salehi F, Al-Gazali L, Hennekam EA, et al. Mutations in CCBE1 cause generalized lymph vessel dysplasia in humans. Nat Genet 2009;41:1272-4.

5. Alders M, Al-Gazali L, Cordeiro I, Dallapiccola B, Ga- 
ravelli L, Tuysuz B, et al. Hennekam syndrome can be caused by FAT4 mutations and be allelic to Van Maldergem syndrome. Hum Genet 2014;133:1161-7.

6. Cormier-Daire V, Lyonnet S, Lehnert A, Martin D, Salomon R, Patey N, et al. Craniosynostosis and kidney malformation in a case of Hennekam syndrome. Am J Med Genet 1995;57:66-8.

7. Bellini C, Mazzella M, Arioni C, Campisi C, Taddei $\mathrm{G}$, Toma $\mathrm{P}$, et al. Hennekam syndrome presenting as nonimmune hydrops fetalis, congenital chylothorax, and congenital pulmonary lymphangiectasia. Am J Med Genet A 2003;120A:92-6.

8. Al Sinani S, Rawahi YA, Abdoon H. Octreotide in Hennekam syndrome-associated intestinal lymphangiectasia. World J Gastroenterol 2012;18:6333-7.

9. Granzow JW, Soderberg JM, Kaji AH, Dauphine C. Review of current surgical treatments for lymphedema. Ann Surg Oncol 2014;21:1195-201. 